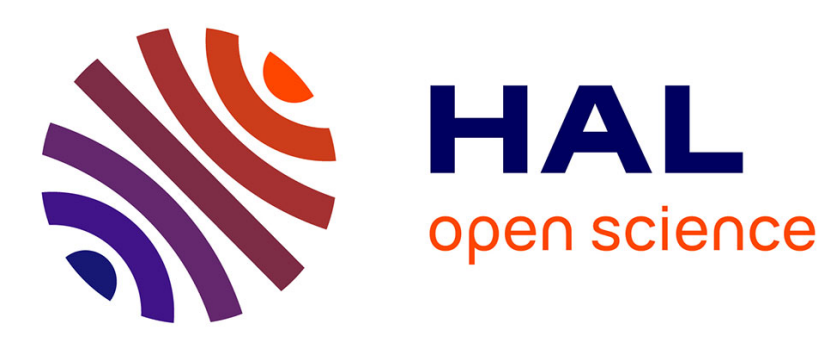

\title{
Après les priorités du jour, le paysage...Enquête sur un enjeu flou pour les services forestiers
}

Philippe Deuffic

\section{To cite this version:}

Philippe Deuffic. Après les priorités du jour, le paysage...Enquête sur un enjeu flou pour les services forestiers. Revue forestière française, 2006, 58 (3), pp.229-244. 10.4267/2042/5996 . hal-03449470

\author{
HAL Id: hal-03449470 \\ https://hal.science/hal-03449470
}

Submitted on 25 Nov 2021

HAL is a multi-disciplinary open access archive for the deposit and dissemination of scientific research documents, whether they are published or not. The documents may come from teaching and research institutions in France or abroad, or from public or private research centers.
L'archive ouverte pluridisciplinaire HAL, est destinée au dépôt et à la diffusion de documents scientifiques de niveau recherche, publiés ou non, émanant des établissements d'enseignement et de recherche français ou étrangers, des laboratoires publics ou privés. 


\title{
ENUIRONNEMENT, cULTURE Et SOCIETE
}

\section{APRÈS LES PRIORITÉS DU JOUR, LE PAYSAGE... ENQUÊTE SUR UN ENJEU FLOU POUR LES SERVICES FORESTIERS}

\author{
Philippe Deuffic
}

Depuis plusieurs décennies, l'impact paysager des aménagements et des travaux forestiers alimente les débats entre gestionnaires et usagers de la forêt. L'Office national des Forêts a retenu la solution du paysagisme d'aménagement, mis en place un réseau de correspondants "paysages" et publié divers guides sur le sujet (Breman et al., 1992 ; Breman, 1993 et 1997 ; ONF, 1999). En forêt privée, des adaptations ont été apportées dans le cas des forêts morvandelles (Breman, 1997) ou limousines (Gernigon, 2002), mais la transposition des techniques du paysagisme d'aménagement s'avérait plus difficile à cause de la structure de la propriété. De fait, tout au long des années 1990, les services forestiers intervenant en forêt privée alertaient périodiquement la Direction de l'Espace rural et de la Forêt (DERF(1) à propos de la persistance de problèmes paysagers. Or, celle-ci est longtemps restée sans véritable politique paysagère (Luginbühl, 1993). Ces questions de paysages étaient d'autant plus difficiles à aborder que les acteurs institutionnels chargés de la forêt et du paysage étaient dispersés entre différents ministères, que les forestiers avaient des difficultés à saisir les attentes des usagers et à intégrer la dimension sociale de la notion de paysage. De plus, forestiers et paysagistes avaient du mal à se comprendre faute de langage et de culture techniques communs. Enfin, les formations initiales et continues étaient souvent inadéquates notamment sur le volet social et culturel de la notion de paysage (Deuffic, 1996). La DERF a essayé de répondre en partie à ces questions en proposant des cycles de sensibilisation au paysage à l'attention des personnels forestiers intervenant en forêt privée. Ces cycles, appelés "Formations Forêts-Paysages", ont eu lieu de 1996 à 2000 et ils ont concerné une cinquantaine d'agents de DDAF, de CRPF et de SERFOB.

En 2002, la DGFAR (ex-DERF) a souhaité faire un nouveau point sur les attentes des agents forestiers intervenant en forêt privée en matière de paysages et réaliser un bilan des "Formations Forêts-Paysages". Cet article présente les principaux résultats de cette enquête notamment les enjeux paysagers tels que les identifient aujourd'hui les agents des services forestiers en charge de la forêt privée, leurs actions et leurs attentes.

\section{LE PAYSAGE, UNE MISSION PARMI D’AUTRES}

\section{Objectifs, méthodes et profils des enquêtés}

L'objectif de l'enquête est de voir quelles sont les questions identifiées par les agents des services déconcentrés autour de la problématique du paysage (en forêt et hors forêt), quelles

(1) La DERF est aujourd'hui devenue la Direction générale de la Forêt et des Affaires rurales (DGFAR). 
difficultés ils rencontrent pour y répondre et quelles sont leurs attentes en termes de moyens et d'outils. Le questionnaire de cette enquête quantitative s'articule autour de trois thèmes : la façon dont les questions de paysage se posent aux agents dans leur pratique professionnelle quotidienne, les outils et les moyens dont ils souhaitent disposer à l'avenir pour pouvoir aborder ces questions, le bilan des formations "Forêts-Paysages" pour ceux qui les ont suivies. Le questionnaire contient essentiellement des questions fermées mais les enquêtés ont la possibilité de rajouter des commentaires libres afin d'expliciter les raisons de leur choix.

135 questionnaires ont été envoyés en octobre 2002 et décembre 2002 dans les services "ForêtEnvironnement" de 95 DDAF, de 22 SERFOB et de 18 CRPF. 69 organismes sur 135 ont répondu, soit un taux de réponse de $51 \%$. Parmi ceux-ci, on trouve 46 DDAF, 14 CRPF et 9 SERFOB. Sur les 109 personnes qui ont renvoyé le questionnaire, 71 travaillent en DDAF (65\% de l'échantillon), 27 en CRPF ( $25 \%$ ) et 11 en SERFOB (10\%). $51 \%$ des personnes qui ont répondu sont des techniciens, $35 \%$ des ingénieurs, $7,5 \%$ des agents et $5 \%$ des chefs de service. Contrairement aux résultats obtenus par Crignon et Mulot (2001), on n'observe pas de corrélation nette entre les taux de réponse et les taux de boisement des départements ou des régions concernés. En revanche, pour un même échelon comme la région, les personnels des CRPF ont été plus nombreux à répondre que ceux des SERFOB. Cette différence peut s'expliquer par une inscription territoriale plus dense des CRPF et par la nature des missions respectives de ces deux organismes pour qui la notion de paysage n'a pas forcément la même importance.

\section{La priorité aux missions régaliennes puis à l'environnement}

Avant d'évoquer en détail la problématique du paysage, nous avons voulu cerner les missions prioritaires aux yeux des agents et replacer ainsi les questions de paysage et leur importance dans le contexte de travail quotidien d'un service forestier décentralisé.

Pour plus de $54 \%$ des agents interrogés, la mission prioritaire ${ }^{(2)}$ relève des activités régaliennes définies dans le Code forestier. Celles-ci les occupent de 50 à $80 \%$ de leur temps. Les agents de DDAF mentionnent essentiellement l'instruction des dossiers de demandes de subventions et le suivi des réglementations (boisement, loi Sérot). Les agents de CRPF évoquent leurs actions

\section{FIGURE 1 MISSIONS PRIORITAIRES ET AUTRES MISSIONS}

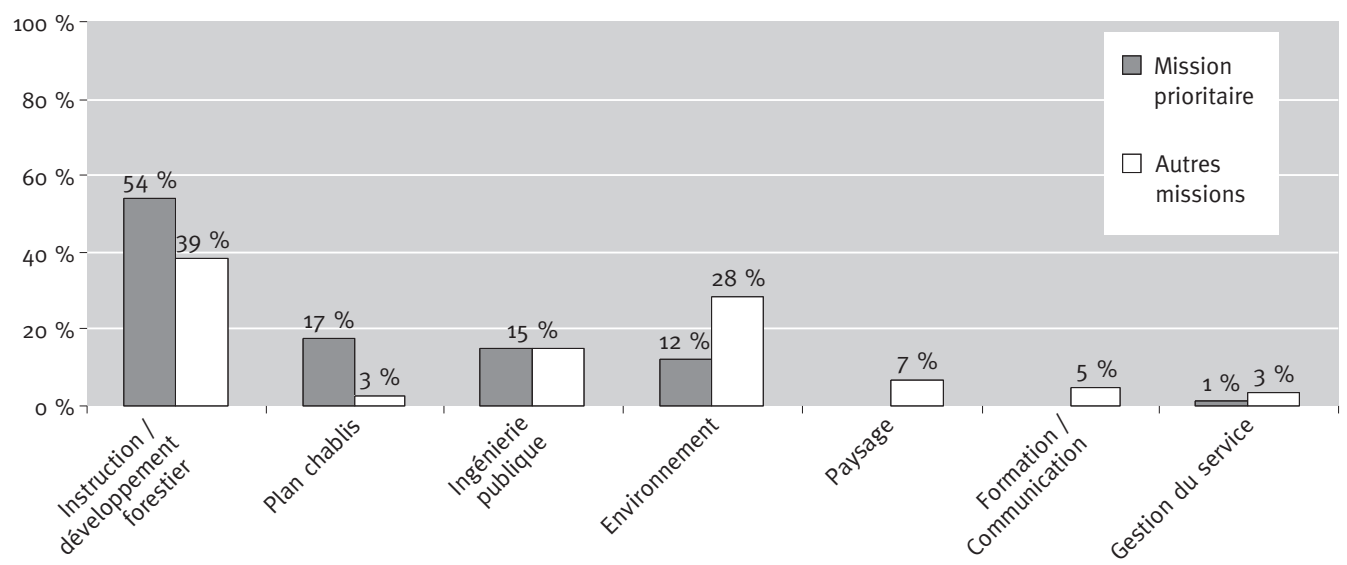

(2) Dans les rares cas où la personne affiche plusieurs missions prioritaires (au lieu d'une), nous avons retenu celle pour laquelle l'agent déclare consacrer le plus de temps. De fait, deux tiers des agents déclarent au moins une mission dite prioritaire ; celle-ci occupe de 50 à $80 \%$ de leur temps de travail. 
en matière de développement et de vulgarisation forestière, l'agrément et le suivi des PSG. Quant aux agents de SERFOB, ils placent en priorité la coordination et la gestion des crédits forestiers, les actions de contrôles et le suivi des contentieux.

Pour $17 \%$ des agents (essentiellement de DDAF), la mission prioritaire actuelle consiste à mettre en œuvre le plan "chablis" suite à la tempête de 1999. 15 \% des enquêtés effectuent en priorité des missions d'ingénierie telles que la mise en place d'équipements forestiers (desserte forestière ou DFCl) ou des opérations de remembrement et d'aménagement foncier. $12 \%$ des agents ont pour priorité des missions à caractère environnemental en majorité reliées à la chasse et à la gestion de la faune sauvage. Aucun enquêté n'a fait allusion au paysage d'une manière ou d'une autre comme faisant partie de ses missions prioritaires.

Concernant leurs "autres missions", la plupart des agents en ont cité plusieurs (de 2 à 5), ce qui montre la diversité des tâches à réaliser. Les agents estiment que ces "autres missions" occupent 20 à $40 \%$ de leur temps de travail.

Les missions régaliennes n'ont pas toutes forcément le même caractère d'urgence et de priorité. C'est pourquoi elles représentent encore le pourcentage le plus important des "autres missions" (39\% des enquêtés ont formulé des réponses relevant de cette catégorie), devant les dossiers à caractère environnemental $(28 \%)$ et les missions d'ingénierie (15\%). Un point significatif de ces "autres missions" est l'importance des dossiers ou des opérations à caractère environnemental, cités 41 fois et qui consistent surtout à suivre la procédure Natura 2000 et les négociations autour de la rédaction des documents d'objectifs (DOCOB). Les actions à caractère paysager sont citées 10 fois (7\% des citations) et concernent $10 \%$ des agents. Ces actions entrent dans le cadre de l'application de la loi "paysage" de 1993, de l'élaboration de chartes architecturales et paysagères, d'atlas de paysages, d'un observatoire photographique. Elles touchent aussi au volet paysager des grandes infrastructures (lignes électriques, TGV, projets autoroutiers).

\section{Le paysage, une importance toute relative}

Tous types de missions confondus, nous voulions savoir si les agents considéraient le thème du paysage comme central, annexe ou négligeable. Les agents ont répondu de deux manières différentes.

56 agents ont considéré l'importance de la notion de paysage de manière globale toutes missions confondues. Pour $62 \%$ d'entre eux, cette notion est annexe quel que soit le type de dossier considéré. Sans l'oublier totalement, c'est une thématique à laquelle ils n'accordent qu'une attention relative, selon les dossiers ou leur sensibilité personnelle : "la prise en compte n'est pas systématique dès lors que l'on n'est pas en zone sensible réglementairement ce qui n'empêche pas de le faire par sensibilité personnelle et de tenter de vulgariser cette donnée auprès des différents acteurs ( $\mathrm{n}^{\circ}$ 89, DDAF) ». Pour $21 \%$ des agents de ce groupe, cette notion est négligeable quel que soit le type de dossier. Seuls $16 \%$ la considèrent comme centrale; pour eux, le paysage fait partie des critères de gestion durable de la forêt. C'est un sujet de discussion qui n'est pas purement technique et qui permet d'évoquer plus largement la place de la forêt dans l'espace rural : " [le paysage], c'est l'entrée principale de tout dialogue avec les acteurs locaux, puisqu'en dehors de toutes contraintes techniques, c'est ce qu'ils appréhendent le mieux ( $\left.{ }^{\circ} 98, \mathrm{DDAF}\right) »$.

L'autre moitié des enquêtés (52 agents) ne porte pas un avis global sur l'importance du thème "paysage" mais établit une hiérarchie fondée sur le type de dossier ou d'opération.

La question du paysage s'avère centrale dans les cas où il existe une protection réglementaire forte (site classé, réserve naturelle, espaces boisés classés). La nature exceptionnelle et pérenne 
des opérations sylvicoles est également essentielle : plus les travaux sont importants en surface et dans le temps et plus cela affecte l'intégrité de la forêt et induit des changements d'usage du sol (cas des coupes rases, des défrichements et des boisements), plus les agents se disent attentifs au contexte paysager. Enfin, la localisation géographique des opérations leur paraît importante surtout quand celles-ci ont lieu dans des secteurs protégés ou des "zones à fort impact visuel ou très fréquentées ( ${ }^{\circ} 10$, DDAF) ".

La prise en compte du paysage est jugée comme "annexe" dans le cas où les opérations portent sur des zones géographiques sans enjeux ou conflits clairement identifiés ou sur la gestion courante des boisements de production (reboisement après coupe rase, éclaircies, dépressages).

Enfin, ces agents considèrent le thème du paysage comme négligeable dans les dossiers traitant d'opération de balivage, de reconstitution des boisements à l'identique ou de peuplements irréguliers.

\section{Espaces et acteurs concernés}

Si $37 \%$ des agents limitent les questions de paysage aux espaces strictement forestiers, $32 \%$ positionnent les enjeux paysagers à l'échelle plus globale de l'espace rural, évoquant les relations agriculture/forêt, le boisement des terres agricoles, la réglementation des boisements, la fermeture des paysages, etc. Certains agents soulignent aussi le rôle paysager des arbres hors forêt (plantations de haies, arbres d'alignement, vergers, agroforesterie...). Ils montrent ainsi qu'il leur est difficile de considérer la forêt indépendamment des autres composantes du paysage rural. Ils le constatent à propos du rôle que la forêt joue dans le territoire et notamment des demandes récurrentes pour "une forêt-loisir ", propice " à un tourisme de nature " et participant « au cadre de vie et au développement touristique ( $\left.{ }^{\circ} 23, \mathrm{CRPF}\right) »$.

Pour la moitié des enquêtés, c'est le rôle social et environnemental de la forêt qui est interrogé à travers les questions de paysage, ce qui génère pour deux tiers des enquêtés des rapports de force et des conflits. Ceux-ci sont tantôt le fait de propriétaires forestiers " qui n'ont pas du tout intégré cette problématique du paysage ( $\mathrm{n}^{\circ}$ 17, DDAF) », tantôt le fait d'associations de protection de la nature ou d'usagers de la forêt. Plusieurs agents mettent aussi en avant le fait que ces " conflits sont fortement exagérés par les médias ( $n^{\circ} 7$, SERFOB) » et parlent même " d'affaires médiatico-politiques ( $\left.n^{\circ} 33, \mathrm{CRPF}\right)$ » ou de dossiers " politisés ( $\left.\mathrm{n}^{\circ} 46, \mathrm{DDAF}\right)$ ». Plus généralement, les agents enquêtés mettent en avant les conflits entre propriétaires ruraux et citadins non propriétaires. Ceux-ci naîtraient des décalages de représentations et de la "vision idéalisée du paysage » véhiculée par les urbains.

Plus de $80 \%$ des agents considèrent également que les questions de paysage forestier ne devraient pas seulement concerner les espaces boisés protégés à titre réglementaire $(86 \%$ de non) ou les forêts publiques ( $90 \%$ de non), ni uniquement les propriétaires forestiers privés ( $90 \%$ de non) mais l'ensemble des usagers de la forêt ( $80 \%$ de oui). On constate toutefois une légère différence selon le service auquel appartient l'agent. Si $83 \%$ des enquêtés travaillant en DDAF pensent que tous les usagers de la forêt sont potentiellement concernés par les enjeux liés au paysage forestier, ils sont $65 \%$ à être de cet avis au sein des CRPF.

Derrière cette relative unanimité sur le type d'espace et d'acteurs concernés, des agents soulignent les difficultés à faire discuter ensemble les propriétaires, les usagers et les représentants des collectivités. Ils s'interrogent sur la représentativité et les motivations de ces différents groupes : "le thème du paysage est essentiellement accaparé par certaines catégories d'usagers mais ne semble pas concerner les propriétaires ni même les populations locales ( $n^{\circ} 74$, DDAF) ». Certains regrettent que "le paysage, qui était autrefois le résultat d'une gestion d'acteurs 
économiques de l'espace, est ou devrait être aujourd'hui le résultat esthétique [à l'attention] de spectateurs passifs ( ${ }^{\circ} 6$, DDAF) ». Une autre interrogation récurrente concerne les coûts éventuellement engendrés par les actions paysagères et leur prise en charge. Les agents envisagent deux pistes : soit une participation collective de l'ensemble des acteurs concernés (État, collectivités, propriétaires et usagers), soit une participation ciblée selon «le principe "prescripteurpayeur" ( $\left.\mathrm{n}^{\circ} 20, \mathrm{CRPF}\right) »$.

\section{ACTIONS RÉALISÉES ET DIFFICULTÉS}

\section{Nature des actions réalisées}

Un autre objectif du questionnaire est d'identifier les tâches à caractère paysager effectuées par les enquêtés. $45 \%$ d'entre eux identifient souvent ou occasionnellement des situations potentielles de conflit de nature paysagère. $38 \%$ instruisent couramment ou occasionnellement des dossiers à caractère paysager qui relèvent le plus souvent d'un cadre réglementaire [loi de 1930 sur les sites classés, agrément des plans simples de gestion (PSG) et des plans locaux d'urbanisme (PLU), autorisation de défrichement, installations classées pour la protection de l'environnement].

\section{FIGURE 2 LES ACTIONS DES ENQUÊTÉS EN MATIÈRE DE PAYSAGE}

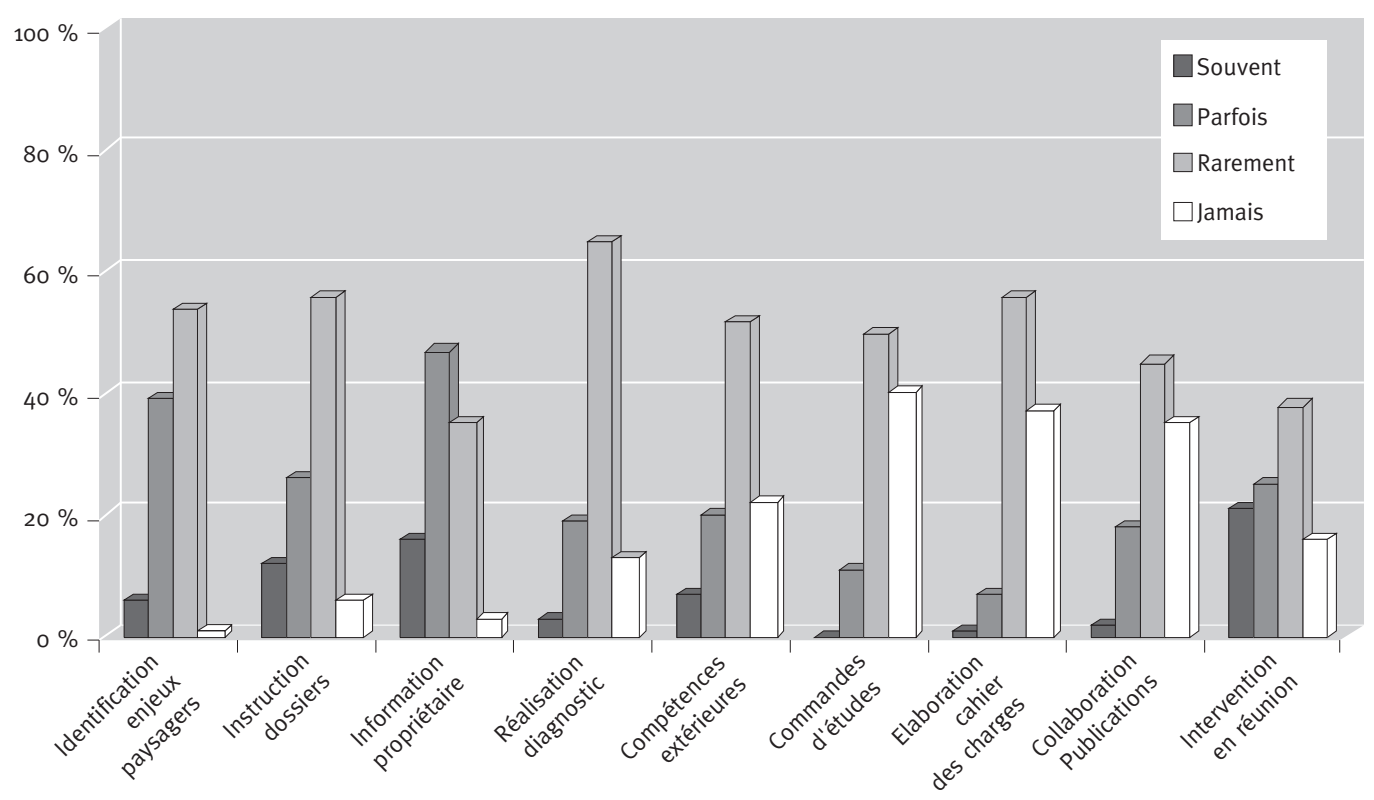

Une majorité des enquêtés (62 \%) informe souvent ou occasionnellement les propriétaires sur les enjeux liés à la prise en compte du paysage à l'occasion de la révision du PSG et pour des opérations sylvicoles importantes (équipement, boisement, conversion). Ces conseils tiennent en des suggestions techniques normatives telles que "ne pas fermer les paysages et éviter les coupes rases de plus de quatre hectares ", "respecter les abords des cours d'eau ", " maintenir un rideau d'arbres devant un chantier de plantation", "implanter des feuillus à la place de résineux »... 
$65 \%$ des enquêtés déclarent rarement réaliser des diagnostics paysagers ; $13 \%$ n'en réalisent jamais par manque de temps, de compétences ou à cause de leur coût. Si $72 \%$ des agents font rarement voire jamais appel à des compétences extérieures, cela est un peu moins vrai pour les agents formés. Ces avis extérieurs sont recueillis auprès d'autres services publics (DIREN, Service départemental d'architecture, CAUE (3)). L'appel aux bureaux d'études reste exceptionnel ; seuls $11 \%$ des agents y ont recours et de manière très sporadique. Ils ont donc peu l'occasion de rédiger des cahiers des charges à leur intention.

Les agents interrogés contribuent exceptionnellement à la réalisation " d'atlas des paysages forestiers ", de "référentiels paysagers ", de "cahiers de recommandation » (cas du Morvan), et à diverses plaquettes de sensibilisation et fiches techniques. Ils sont associés à ces projets pour leurs compétences forestières, la partie strictement paysagère relevant souvent d'un autre intervenant.

Si $21 \%$ des agents s'estiment relativement à l'aise pour aborder les questions de paysage en réunion publique, certains agents relèvent que le caractère subjectif de cette notion rend plus complexes les discussions. Les uns se servent donc de "la subjectivité " pour relativiser toute appréciation portée sur un dossier ou une opération, estimant que le point de vue exprimé n'est que le reflet d'un avis individuel ou d'un groupe marginal. D'autres ignorent cette dimension par crainte de ne jamais parvenir à une synthèse ou un consensus opérationnel. Il y a enfin ceux qui s'en accommodent et pour qui "le concept de subjectivité ne conduit pas nécessairement à des conflits ( $n^{\circ} 7$, SERFOB) ».

\section{Difficultés rencontrées}

Parmi les difficultés auxquelles les agents sont confrontés dans leur tentative de prise en compte du paysage, les enquêtés invoquent :

- le manque d'intérêt des propriétaires pour ces questions : pour $77 \%$ des enquêtés, ces questions de paysage seraient considérées comme mineures par les propriétaires avec lesquels ils travaillent : "vu le contexte économique difficile, les propriétaires considèrent très souvent $q u^{\prime} i l s$ ne peuvent pas tout assumer, et, en ce sens, le paysage n'apparait pas prioritaire $\left(n^{\circ} 23\right.$, CRPF) ». Cependant les agents ont le sentiment que cette indifférence des propriétaires s'atténue et qu'ils participent, par leur travail quotidien, à cette prise de conscience : " les propriétaires ne se posent pas la question du paysage a priori mais ils sont globalement réceptifs lorsqu'on leur montre les enjeux à long terme ( $\left.n^{\circ} 81, \mathrm{CRPF}\right)$ ";

- la polysémie de la notion : $70 \%$ des agents enquêtés estiment que les discussions sur le thème du paysage sont difficiles à mener. La principale raison tient à la subjectivité de la notion qu'ils n'arrivent pas à intégrer dans leur analyse. Cependant, quelques agents n'admettent pas que l'on réfute toute problématique à caractère paysager au prétexte de la polysémie de cette notion : "l'approche subjective du paysage est l'argument tarte à la crème de ceux qui ne veulent pas discuter. Lorsque les partenaires sont ouverts à une discussion et à une éventuelle évolution de leur dossier initial, les choses peuvent avancer sur le fond ( $n^{\circ} 17$, DDAF) ";

- des orientations institutionnelles floues : $53 \%$ des enquêtés jugent les orientations institutionnelles floues en matière de paysage même s'ils les comprennent dans une certaine mesure. En effet, la question du paysage leur paraît trop dépendante du contexte régional et local pour être formalisée dans des directives ou des circulaires nationales. Certains souhaiteraient tout au plus que le contenu des diagnostics paysagers soit plus explicite. D'autres se satisfont de ce flou réglementaire ; ils préfèrent adapter et préciser les enjeux de paysage au niveau des orientations

(3) Conseil en architecture, urbanisme et environnement. 
FIGURE 3 LES DIFFICULTÉS DES ENQUÊTÉS EN MATIÈRE DE PAYSAGE

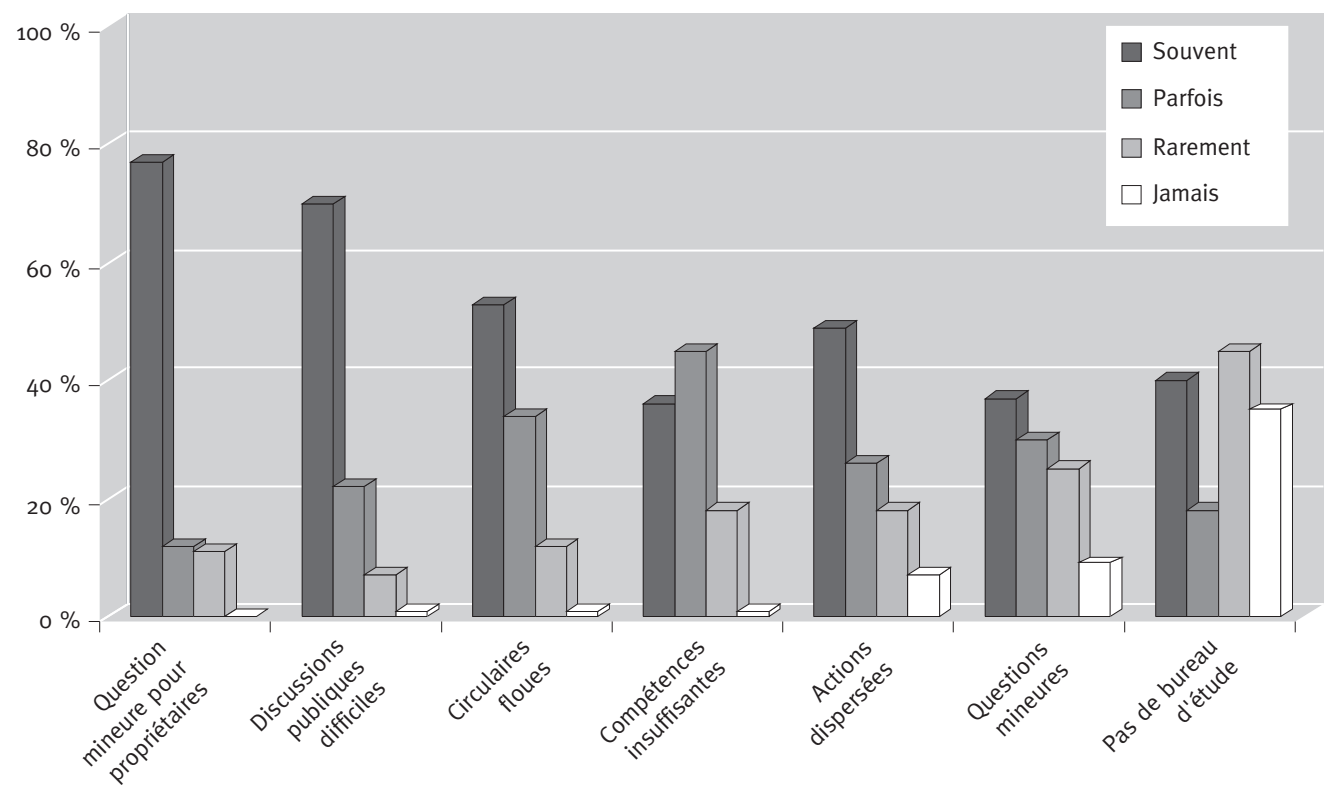

régionales de production et "laisser plus de place à la concertation sur le terrain $\left(\mathrm{n}^{\circ} 75\right.$, DDAF) »;

- un niveau de compétence insuffisant : $17 \%$ des enquêtés considèrent que leur niveau de compétence n'est pas un obstacle à la prise en compte du paysage et qu'un peu de "bon sens " suffit pour répondre à ces questions. À l'inverse, $36 \%$ des enquêtés pensent que leurs compétences sont insuffisantes et que le bon sens a ses limites : "mes compétences sont insuffisamment étayées et reconnues : la production, c'est la DDAF ; le paysage, c'est la DIREN. Et, au-delà du bon sens, il faut avoir le langage adapté ( $n^{\circ} 37$, DDAF) ». La moitié des enquêtés considèrent prosaïquement que leur niveau de compétence est " parfois » un frein, selon les dossiers, leur nature et l'ampleur du problème posé ;

- une préoccupation mineure : un tiers des enquêtés estime que les questions de paysage sont secondaires. Deux raisons sont avancées : soit le manque de temps et la gestion des priorités, soit l'absence de motivation. A contrario, un quart d'entre eux estime que le paysage n'est pas une question mineure et que le prendre en compte peut permettre " d'éviter des conflits potentiels entre usagers de la forêt ( $n^{\circ} 46$, DDAF) »;

- des compétences difficiles à trouver : $40 \%$ des enquêtés déclarent avoir des difficultés à trouver des professionnels ayant une double compétence (paysagère et sylvicole). Soit ils jugent les paysagistes incompétents et ils les accusent « de raconter n'importe quoi sur la forêt et de n'avoir aucune solution technique concrète ( $n^{\circ} 31$, SERFOB) ». Soit ils n'en connaissent pas et ne savent pas à qui s'adresser : "si un dossier très sensible se présentait, je ne suis pas sûr de pouvoir indiquer des références de bureau d'études aux maîtres d'œuvre et d'ouvrage $\left(\mathrm{n}^{\circ} 46\right.$, DDAF) ». À défaut de paysagistes, certains enquêtés se tournent vers les experts forestiers. Outre le fait que ceux-ci soient peu nombreux à être formés, cette attitude dénote une méfiance vis-àvis des professionnels du paysage et une tendance à privilégier les acteurs appartenant au même univers technico-professionnel qu'eux ;

- la question des coûts : quelques remarques ont été formulées à propos du coût des aménagements paysagers. Selon cet ingénieur de CRPF, « la prise en compte du paysage (...) 
doit faire l'objet d'une contractualisation intégrant la notion de rémunération du service dès lors que ce service dépasse la simple application des règles et des itinéraires de gestion sylvicole normale ( ${ }^{\circ} 33$, CRPF) ». Un confrère enfonce le clou en déclarant que "dans ce dossier, il manque deux choses : un outil simple d'estimation de la qualité d'un paysage et des moyens de rémunérer ceux qui produisent ce paysage (n 63, CRPF)".

\section{Atouts et moyens à disposition}

Un des moyens les plus simples pour les agents enquêtés d'aborder ces questions de paysage, c'est la formation "sur le tas". En effet, moins de $10 \%$ des agents estiment que leur formation initiale leur permet d'aborder ces questions, souvent faute d'un enseignement spécifique sur ce thème [sauf dans les cas particuliers de quelques agents ayant suivi un brevet de technicien supérieur (BTS) "gestion et protection de la nature" ou sortis récemment de la $\mathrm{FIF}^{(4)}$ ]. Pour $80 \%$ d'entre eux, la confrontation à la problématique paysagère se fait surtout par l'apprentissage direct sur le terrain, le contact avec les professionnels ou par la formation continue, voire les trois ensemble.

Concernant la formation continue, on constate une différence significative entre agents formés et non formés. 85 \% des agents ayant suivi les formations "Forêts-Paysages" proposées par le ministère de l'Agriculture entre 1996 et 2000 considèrent que la formation continue les aide beaucoup ( $52 \%$ ) ou un peu (33\%) (contre respectivement 20 et $25 \%$ pour les agents non formés). Ce type d'outil semble donc un moyen pertinent d'augmenter leurs compétences à condition de trouver les stages adéquats, traitant simultanément les deux thématiques sylvicoles et paysagères. Outre les formations "Forêts-Paysages", certains agents ont ainsi suivi les formations proposées par l'ENITA (5) de Clermont-Ferrand et l'ENGREF ${ }^{(6)}$, par la cellule "Paysage" de l'ONF ou par des structures locales (CPIE ${ }^{(7)}$, cellule "Forêt-Paysage" du Limousin, FVFE ${ }^{(8)}$ ). Aucun agent ne semble avoir suivi de formations organisées par les écoles de paysages (ENSP, INH, ENSAPB ${ }^{(9)}$...). Cela renforce le sentiment d'un manque de connexion entre le monde de la formation professionnelle forestière et celui des paysagistes. Toutefois, depuis quelques années, l'accent est mis, de part et d'autre, sur la formation initiale des élèves forestiers au paysage et des étudiants paysagistes à la sylviculture (Freytet et Rumelhart, 2001).

D'autres outils leur paraissent plus ou moins utiles. S'ils jugent les orientations réglementaires ${ }^{(10)}$ floues et de portée trop générale, elles n'en sont pas moins utiles en tant qu'outil de légitimation de l'action des agents lors de l'instruction de dossiers comportant des enjeux paysagers : "le thème du paysage abordé dans les circulaires et les rapports d'instruction facilite pour nous les recours, auprès des propriétaires, au nom de la prise en compte de la notion du paysage $\left(n^{\circ} 55\right.$, DDAF) ». Parallèlement, la mise en place de dispositifs incitatifs atténue le côté strictement réglementaire qu'il s'agisse des aides à la plantation de feuillus ou du financement des études paysagères. La notion de paysage est alors " d'autant plus facile à faire passer, comprendre et adopter qu'il y a une carotte (subventions) ou le bâton (sanction réglementaire) à la clé (nº 92, DDAF) ».

(4) FIF : Formation des Ingénieurs forestiers de l'ENGREF à Nancy.

(5) ENITA : École nationale des Ingénieurs des Travaux agricoles.

(6) ENGREF : École nationale du Génie rural, des Eaux et des Forêts.

(7) CPIE : Centre permanent d'Initiatives pour l'Environnement.

(8) FVFE : Fédération de la Vulgarisation forestière de l'Est.

(9) ENSP : École nationale supérieure du Paysage ; INH : Institut national d’Horticulture ; ENSAPB : École nationale supérieure d'Architecture et du Paysage de Bordeaux.

(10) Les circulaires les plus fréquemment citées sont les circulaires DERF/SDF/C2000-3021 du 18 août 2000, C3010 du 7 mai 2001, C3022 du 31 août 2000 et les arrêtés régionaux sur les aides aux investissements forestiers. 
FIGURE 4 LES ATOUTS DES ENQUÊTÉS EN MATIÈRE DE PAYSAGE

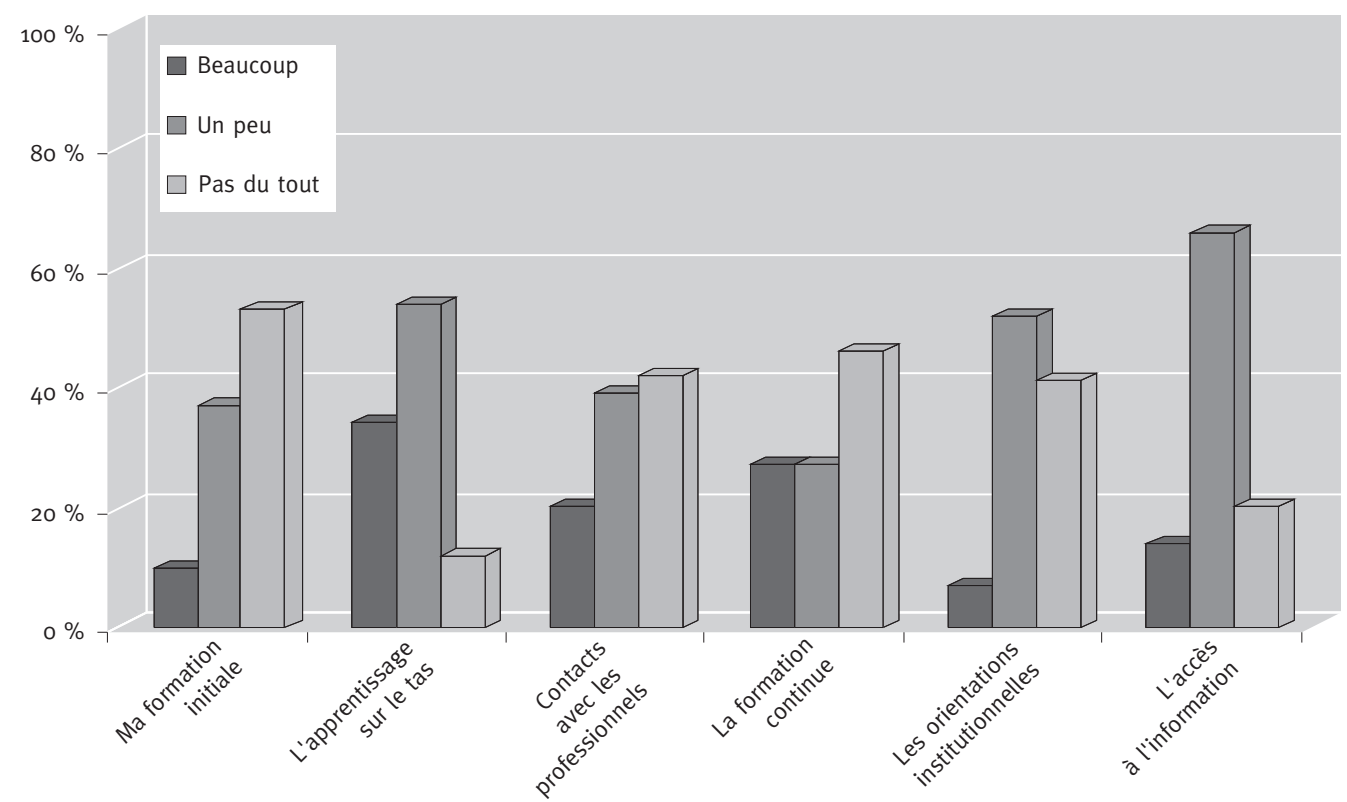

Si deux tiers des agents estiment accéder assez facilement à l'information, celle-ci leur paraît en revanche dispersée ; ils ont l'impression d'aller à la pêche, " de glaner des articles au hasard des revues qui circulent dans le service ( $n^{\circ}$ 64, DDAF) ». Parmi ces revues, ils citent avant tout les revues de vulgarisation telles que Arborescence ${ }^{(11)}$, Forêt-Entreprise, La Forêt privée, Forêts de France. Quelques agents disent aussi ne pas trouver les renseignements suffisants dans les ouvrages sur les paysages ruraux car ceux-ci traitent abondamment des modèles d'aménagements paysagers appliqués à l'espace agricole et plus rarement des exemples d'aménagements paysagers en forêt. De fait, les agents citent plus volontiers les ouvrages spécifiquement dédiés au paysage forestier (Cemagref, 1981 ; Breman, 1993 et 1997 ; Gernigon, 2002).

\section{PERSPECTIVES DU POINT DE VUE DES ENQUÊTÉS}

La troisième partie du questionnaire visait à cerner les perspectives des agents en matière d'investissement personnel sur cette problématique du paysage et les moyens qu'ils souhaitaient voir mettre en œuvre.

\section{Le paysage, une préoccupation en devenir}

Si la problématique du paysage est présente de façon marginale dans les dossiers ou missions des agents, 82 \% d'entre eux s'attendent néanmoins à être de plus en plus souvent confrontés à cette question dans les années à venir. Pour ce forestier de l'Est de la France (nº 66, CRPF), "le paysage devient l'une des composantes de la gestion durable des forêts " au même titre que la biodiversité. Pour cet autre agent, ce mouvement n'ira qu'en s'amplifiant vu la demande

(11) Cette revue a disparu ; elle est remplacée par deux autres revues toujours éditées avec le concours de, ou par l'ONF Arbres et Forêts et Rendez-vous techniques. 
sociale autour de cette thématique : "Le paysage forestier prendra une place de plus en plus importante car le public en général y est de plus en plus sensible. C'est donc, pour nous, forestiers, en particulier lors de l'attribution d'aides publiques, une responsabilité de plus en plus grande qui s'exerce sur du long terme ( $n^{\circ}$ 9, DDAF) ». D'autres enquêtés, plus mitigés, ne voudraient pas que l'on agisse au nom du paysage partout et à tout propos : " Il ne faut pas, à mon avis, que le thème du paysage prenne une dimension démesurée dans le traitement des dossiers. Les sensibilités paysagères ne sont pas équivalentes selon les secteurs géographiques $\left(n^{\circ} 74, \mathrm{DDAF}\right) »$.

Selon les personnes enquêtées, certaines problématiques méritent une attention particulière notamment à cause de leur dimension paysagère :

- le boisement naturel ou artificiel des terres agricoles et l'équilibre agriculture/forêt ( $21 \%$ des citations). Les agents s'interrogent sur l'avenir des terres agricoles en déprise, sur l'opportunité de recourir systématiquement au boisement pour pallier l'enfrichement et sur le rôle que la forêt pourrait jouer en matière de développement local. Sur le plan paysager, ils craignent une "fermeture des paysages » et une complexification de la gestion sylvicole vu le morcellement et l'hétérogénéité des boisements. Pour l'instant, ils interviennent plutôt à la marge, sur le sens des lignes de plantation, le choix des essences, le regroupement des parcelles, plus rarement sur l'opportunité même du boisement ;

- la prise en compte du paysage dans les chartes forestières de territoires, les chartes de parcs naturels régionaux, les chartes architecturales et paysagères (16 \%). Leurs attentes concernent notamment la façon d'inscrire la thématique du paysage dans ce type de documents (comment se positionner : en rester à des principes généraux ou édicter des recommandations précises ? etc.) ;

- les techniques d'intégration paysagère des travaux forestiers courants (15\%) ainsi que ceux menés à l'occasion des chantiers de reconstitution après la tempête ( $14 \%$ des citations) : dans ce cas, les questions portent sur le choix et les associations d'essences, la création d'une zone tampon, la suppression de micro-boisements ou sur les moyens de limiter l'impact paysager d'un défrichement, d'un reboisement, d'une éclaircie, d'une desserte ou d'une infrastructure de $\mathrm{DFCl}$, etc.

\section{Attentes en termes de savoirs, d'outils et de moyens}

Concernant les besoins des agents en termes de savoirs, d'outils ou de moyens, plus de $35 \%$ d'entre eux déclarent ne manquer de rien, soit parce qu'ils ne souhaitent pas s'investir sur cette question, soit parce que rien de ce qui leur est proposé ne les intéresse.

$39 \%$ des agents attendent avant tout du temps et des moyens humains supplémentaires dans le cadre de leur service ( $49 \%$ des citations). Nombre d'entre eux estiment qu'ils n'ont " plus les moyens de remplir leurs missions " et qu'il leur est "impossible d'approfondir le moindre dossier (n 88, DDAF) ». Selon plusieurs enquêtés, cela s'est aggravé depuis la tempête de 1999 au point d'être amené à " traiter les dossiers de reconstitution à la chaîne sans aucune réflexion sur le bien-fondé des options choisies par le demandeur ( ${ }^{\circ}$ 97, DDAF) ». De fait, des agents disent qu'ils n'aborderont ces questions de paysage que "lorsque ce dossier sera plus prioritaire que les multiples priorités du jour ( $n^{\circ} 17$, DDAF) ». Cette question des moyens humains est récurrente. Elle révèle le malaise des agents des services forestiers de DDAF qui ont le sentiment confus d'être le parent pauvre des différents services des Directions départementales de l'Agriculture et de la Forêt. Ils se demandent s'ils font partie d'un "service" ou seulement d'une "cellule Forêt" intégrée dans un ensemble plus vaste qui aborde aussi les questions de chasse, de pêche, d'hydraulique et d'environnement. Ces interrogations portent aussi sur ce qui constitue aujourd'hui le cœur de leur métier - la production forestière ou l'environnement, les missions 
régaliennes ou l'ingénierie publique, l'application des réglementations ou la négociation de normes, etc. - et sur les moyens qui leur sont donnés pour l'exercer.

26 \% des enquêtés souhaitent acquérir des connaissances en matière d'analyses paysagères, de diagnostics paysagers et de techniques d'intégration paysagère des opérations sylvicoles. Ils souhaitent également disposer d'outils du type "guides techniques" et d'information à caractère réglementaire pour eux-mêmes mais aussi pour les propriétaires forestiers qui sont, à leurs yeux, les premières personnes à informer et à motiver.

Certains agents rappellent aussi que, même avec une débauche de moyens et d'outils au sein des services forestiers et tout en faisant preuve de motivations, rien ne se fera sans " une réelle appropriation de la notion de paysage par les acteurs et les décideurs et sans un réel consensus sur les objectifs à atteindre ( $\mathrm{n}^{\circ}$ 109, DDAF) ». Comme le souligne cet autre enquêté, le paysage est aujourd'hui " un sujet non prioritaire à court terme malgré un intérêt et une sensibilité personnels assez forts : [c'est une problématique] trop éloignée des préoccupations actuelles des propriétaires forestiers [d'autant qu'il y a] peu sinon pas d'incitations ( $\left.\mathrm{n}^{\circ} 23, \mathrm{CRPF}\right)$ ». Dans le même ordre d'idée, certains agents estiment qu'il faudra à terme " un cadre simple et adapté aux propriétaires pour contractualiser les services paysagers comprenant l'idée simple d'une rémunération du service et pas seulement des primes à l'investissement paysager, ou une indemnité liée à la contrainte de non-production ainsi que des financements à la hauteur des résultats souhaités ( $n^{\circ} 33$, CRPF) ».

\section{Investissements envisagés}

$88 \%$ des 109 agents enquêtés souhaitent, au moins sur le principe, enrichir leur connaissance sur le paysage dans les mois à venir.

La mise à disposition de guides techniques est, de l'avis des enquêtés, le moyen le plus approprié d'enrichir leur connaissance. Ils sont également intéressés par des informations plus informelles via un bulletin, une lettre de liaison voire un site web. Le contenu serait essentiellement tourné vers "le partage d'expérience entre DDAF, DRAF, ONF, CRPF ", la création " d'une base de données sur des expériences déjà tentées [et analysées en termes de] réussites, d'échecs, de motifs ", d'un "forum où les circulaires seraient mises à disposition ainsi que les documents techniques en ligne ».

FIGURE 5 TYPES DE SUPPORT SOUHAITÉ POUR AMÉLIORER SES CONNAISSANCES $(\mathrm{N}=88)$

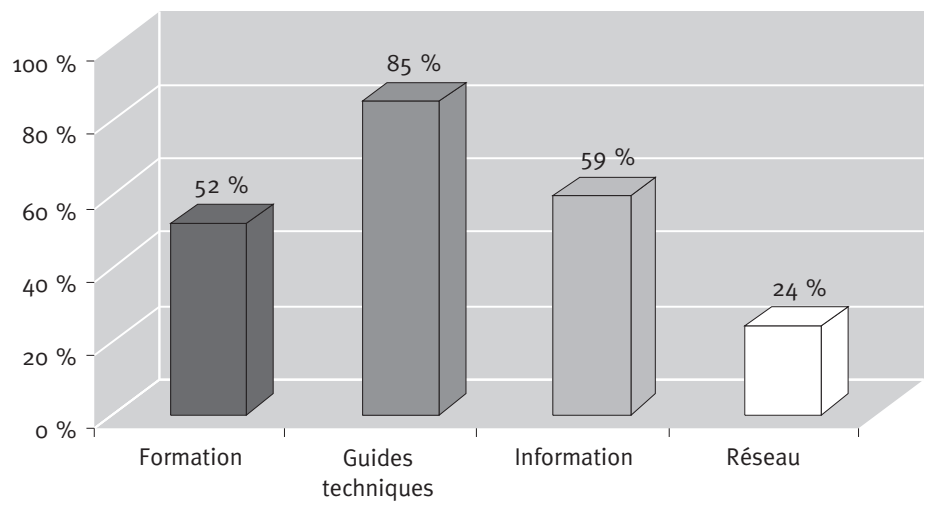


Les réponses concernant la formation continue sont plus mitigées puisque $52 \%$ des agents qui souhaitent approfondir leur connaissance, le feraient par ce biais (soit 46 personnes dont 6 agents déjà formés). Toutefois, le manque de disponibilité en temps reste à leurs yeux une contrainte majeure. Pour cette personne qui travaille dans une région sinistrée par la tempête, "le nombre de dossiers "reconstitution après-tempête" déposés et instruits dans les années 2001 et 2002 est de l'ordre de 320, et, vu les délais d'attentes, je ne peux pas me permettre de dégager du temps pour de la formation ( $n^{\circ} 11$, DDAF) ». Quant aux autres enquêtés, ils ne souhaitent pas que les formations excédent 2 à 3 jours, 5 au maximum. Cependant, certains agents estiment que les formations ne doivent pas non plus être trop courtes car "l'acquisition de compétences sur 2 jours est forcément très limitée ( $n^{\circ} 23$, CRPF) ». Ces formations devraient aborder l'ensemble de la thématique paysagère, c'est-à-dire "la méthodologie, le diagnostic, l'impact des travaux projetés et les mesures compensatoires ( $n^{\circ} 32$, DDAF) » et être organisées " autour d'exposés en salle et d'études de cas particuliers sur le terrain avec des spécialistes du paysage ( $\mathrm{n}^{\circ} 86$, DDAF) ».

Enfin, $76 \%$ des agents préfèrent ne pas participer à un réseau. Outre le fait qu'ils ne souhaitent pas intégrer une telle structure sans être préalablement formés à cette thématique du paysage, certains agents s'appuient sur des expériences précédentes de constitution de réseau qui se sont avérées lourdes ou qui n'ont pas abouti.

\section{CONCLUSIONS}

Cette enquête montre que la mise en œuvre des politiques forestières, la gestion des crédits afférents, l'instruction de dossiers et le conseil à l'attention des propriétaires constituent les missions prioritaires pour une majorité des agents enquêtés. Si les questions d'environnement occupent une place de plus en plus importante, les missions à caractère strictement paysager leur semblent en revanche secondaires. Cela s'explique par la rareté et le caractère peu stratégique des dossiers comportant un volet paysager, par le choix des agents de ne pas investir un domaine sur lequel ils se sentent incompétents ou par la dimension transversale de ces questions de paysage, présentes dans de nombreux dossiers mais rarement de façon centrale.

Quant à l'affirmation quasi unanime des agents de la nécessité d'impliquer sur ces questions de paysage l'ensemble des acteurs et des espaces forestiers - publics ou privés, propriétaires ou usagers - on peut s'interroger sur sa traduction réelle dans leur travail quotidien. En effet, l'importance et l'attention que les agents portent aux questions de paysage semblent d'abord fonction de l'existence d'une réglementation paysagère sur le site considéré, du type d'opérations sylvicoles prévues et de la localisation géographique du projet. De fait, les actions essentielles des agents en matière de paysage consistent à identifier les situations de conflits, à informer les propriétaires et à instruire des dossiers comportant un volet paysager.

Mais la difficulté majeure pour les agents enquêtés reste le désintérêt des propriétaires pour cette thématique, ce qui leur paraît d'autant plus compréhensible que la question des moyens et des contreparties financières est rarement posée et encore moins résolue. Les autres difficultés tiennent à la polysémie et la subjectivité de la notion de paysage, au manque de compétences, à la dispersion des actions des agents, au manque de temps pour approfondir une recherche personnelle... Les agents sont aussi tentés de restreindre la problématique du paysage à sa dimension technique comme le montre leur attente en matière de guides de référence. Ils sont aussi méfiants vis-à-vis des professionnels du paysage. Certains enquêtés souhaitent ainsi voir la question du paysage investie par les experts forestiers plutôt que de voir les paysagistes investir les questions de gestion forestière. Même si l'univers professionnel forestier s'est considéra- 
blement ouvert à d'autres acteurs et d'autres formes de savoir depuis vingt ans, cette méfiance vis-à-vis d'autres corps d'experts est assez commune dans le monde forestier occidental : au Canada (Guay et Gagnon, 1988 ; Wagner et al., 1998), aux États-Unis (Cramer, 1993) et en Europe (Schmithüsen et Kazemi, 1995 ; Dasnoy et al., 2000 ; Kennedy et al., 2001).

Malgré ces difficultés, les agents abordent ces questions de façon pragmatique par l'apprentissage "sur le tas", par la lecture d'ouvrages et d'articles et par le contact direct avec les professionnels. $80 \%$ des agents interrogés estiment que ces questions de paysage se poseront de plus en plus souvent dans les années à venir notamment pour ce qui concerne l'équilibre forêt/agriculture et la question du boisement, artificiel ou naturel, des terres agricoles. La moitié d'entre eux souhaiterait disposer d'outils d'analyse et de méthodologie, de guides techniques mais aussi de temps et de moyens, humains ou financiers, leur permettant, par exemple, de faire appel à des compétences extérieures. Certains souhaiteraient également un cadre contractuel simple permettant de lier "offreurs" et "demandeurs" de biens et de services environnementaux offerts par les espaces forestiers, ce à quoi les chartes forestières de territoire peuvent en partie répondre.

Après cette enquête, plusieurs interrogations restent cependant en suspens. L'une concerne notamment l'évolution des missions et des compétences des agents vis-à-vis de ces questions de paysage. L'enquête montre que les questions de paysage restent marginales et que les agents ne peuvent, sans formation spécifique, aller au-delà de quelques recommandations de base. Pour autant, la définition du futur champ de compétence ne va pas de soi. Sachant que les missions d'ingénierie diminuent, on peut s'interroger sur la pertinence de formations axées sur les techniques d'intégration paysagère. Inversement, les formations au paysage doivent intégrer les sujétions liées à la gestion forestière, y compris celles liées à la sylviculture régulière. Quant au niveau de compétence à acquérir, il peut beaucoup varier allant d’un premier niveau de mémorisation de l'information, à un niveau de compréhension, de maîtrise voire d'innovation et de transfert (Jouvenel et Masingue, 1994). Dans tous les cas, la reconnaissance et la mise en pratique effective des connaissances acquises paraissent nécessaires faute de quoi le bénéfice de ces stages se perd rapidement.

On peut enfin s'interroger sur les difficultés d'accéder à des ouvrages traitant de ce thème. Diverses publications, dont certaines très récentes, condensent des informations spécifiques sur le paysage. Un porter-à-connaissance et une diffusion de ce qui existe déjà devraient satisfaire en partie ces demandes. Malgré cela, le degré d'intervention directe des agents sur cette thématique du paysage est rapidement limité par les autres tâches quotidiennes. Les techniciens forestiers peuvent conseiller un propriétaire et suggérer quelques adaptations paysagères à un projet sylvicole simple et limité en surface et surtout en termes d'enjeux. Mais, dans des cas plus complexes, les agents déclarent disposer de trop peu de leviers notamment financiers pour impulser ou participer au financement d'une action comprenant, au minimum, la mise en place d'un comité de pilotage et son animation, l'intervention d'un professionnel qui réalise une étude approfondie et le suivi de la mise en œuvre du projet. Quand bien même ces moyens existent, le recours à des professionnels du paysage nécessite un réajustement des attentes du forestier car celui-ci a peu de chance de trouver le profil idéal du paysagiste expert en question forestière ; l'idée serait plutôt d'accompagner les paysagistes et de construire avec eux le projet paysager et sylvicole, ce qui nécessite du temps, des confrontations relativement fréquentes et un minimum de langage commun.

Outre la faiblesse de ces "crédits d'étude", la faible contrepartie financière reversée en cas de modification d'un projet sylvicole pour des motifs paysagers (et environnementaux de façon plus générale) constitue un autre frein. Le développement de "contrats" liant offreurs et "demandeurs" de biens et de services offerts par les territoires forestiers paraît être une piste intéressante. 
Il s'agirait de reconnaître financièrement les fonctions environnementales de la forêt sous la forme d'un "contrat de gestion environnementale" entre les pouvoirs publics et les propriétaires (Barthod, 2003). L'approche fiscale et réglementaire ne perdurerait que dans les cas les plus difficiles. L'approche contractuelle présente l'avantage de renforcer le rôle et les responsabilités du gestionnaire en place et de limiter les efforts financiers au coût marginal. Plutôt que la taxation des pratiques défavorables à l'environnement, on inciterait ainsi financièrement les actions positives en matière de gestion durable, d'environnement et de paysage.

\author{
Philippe DEUFFIC \\ UR “Agriculture et dynamique de l'espace rural” \\ CEMAGREF \\ 50, avenue de Verdun \\ F-33612 CESTAS CEDEX \\ (philippe.deuffic@bordeaux.cemagref.fr)
}

\title{
Bilan de la formation "Forêts-Paysages 1996-2000"
}

Sur les 47 agents qui ont suivi la formation, 22 d'entre eux ont répondu au questionnaire. $\mathrm{Si}$ leurs réponses ne sont pas généralisables à l'ensemble des personnes formées vu la faible taille de l'échantillon, elles permettent néanmoins de dégager certaines tendances.

L'évaluation "à froid" de la formation "Forêts-Paysages" est, dans son ensemble, très positive. L'évaluation pédagogique montre que le langage spécifique aux approches paysagères semble acquis et permet aux forestiers de mieux comprendre les paysagistes professionnels. Les stagiaires acceptent aussi plus facilement la dimension sociale et subjective inhérente au paysage et sa prise en compte comme critère de l'analyse paysagère. Ils prennent conscience que, malgré leurs savoirs en matière forestière ou environnementale, ils n'ont pas a priori plus de légitimité que d'autres groupes sociaux pour définir ce que peut être le paysage. Le troisième résultat concerne la démarche pédagogique : le concept de site-atelier est très apprécié par son côté concret et démonstratif. De fait, l'acquisition des techniques d'intégration paysagère des opérations sylvicoles s'avère plus rapide. Concernant l'évaluation professionnelle, l'enquête montre que les stagiaires "Forêts-Paysages" sont proportionnellement toujours plus nombreux à agir que les agents non formés. En outre, leurs capacités d'intervention sont plus larges : il s'agit alors le plus souvent d'informer le propriétaire de l'intérêt de prendre en compte le paysage, de réaliser un diagnostic rapide, à une échelle de territoire restreinte (un massif, une parcelle), d'adapter les techniques sylvicoles classiques en vue d'une meilleure intégration paysagère et convaincre le propriétaire du bien-fondé de ces modifications. En revanche, ils mobilisent peu de compétences extérieures car celles-ci nécessitent des moyens financiers et humains spécifiques et souvent externes au service, ce dont ces agents disposent rarement.

Si les agents formés entre 1996 et 1999 regrettent l'absence de reconnaissance (ou la reconnaissance tardive) de leur effort en matière d'acquisition de nouveaux savoirs, ils continuent néanmoins à s'intéresser à cette problématique du paysage et à considérer que celle-ci doit être prise en compte dans le cadre de la gestion de l'espace rural (en forêt et hors forêt).

Si 15 à $35 \%$ des agents formés estiment que la formation a eu des conséquences directes sur leur travail au quotidien, une partie bien plus importante ne voit pas d'effets directs. Les retombées concrètes d'une formation sont certes difficiles à estimer et très dépendantes de facteurs externes. Néanmoins, la formation continue semble atteindre ici plusieurs de ses limites. Premièrement, si le programme pédagogique paraît adapté, l'application des acquis dans une situation professionnelle semble difficile (manque de reconnaissance au sein du service, pas de temps ni 
de moyens dégagés, priorités faites à d'autres missions...). Ensuite, la formation continue ne peut pas jouer tous les rôles et couvrir à elle seule un champ d'intervention aussi large que celui d'une politique paysagère. La formation initiée par le ministère en charge de l'Agriculture et de la Forêt ne peut être qu'une partie d'un mouvement de réforme beaucoup plus vaste de la politique forestière française tel que celui initié par la loi d'orientation sur la forêt de 2001, la réforme du Fonds forestier national et la mise en place de mesures forestières dans le cadre du Fonds européen agricole de développement rural (FEADER). Enfin, il ne faut pas que la mise en place de cette formation continue pallie un manque de clarté, de sens et de moyens du ministère de l'Agriculture en charge des Forêts sur cette thématique. Tout se passerait alors comme si on répondait à une crise de sens (quelle place pour le paysage dans les politiques forestières ?) par des transformations organisationnelles (comment améliorer les compétences du service sur ce thème du paysage ?). Faute d'horizons de significations bien dégagés sur cette problématique du paysage, il pourrait être tentant de se concentrer sur les manières de faire en guise de compensation procédurale plutôt que sur les raisons d'agir.

\section{BIBLIOGRAPHIE}

AMBROISE (R.), BONNEAUD (F.), BRUNET-VINCK (V.). - Agriculture et paysages : dix exemples de projets de paysage en agriculture. - Dijon : Educagri éditions, 2000. - 207 p.

BARBIER (J.-M.). - Paysage et forêt : un sujet difficile, mais des avancées possibles. - Forêt-Entreprise, vol. 140, 2001, pp. 17-18.

BARTHOD (C.). - La Contractualisation, outil d'avenir des politiques forestières ? In : XII Congrès forestier mondial, FAO, Québec, 2003. - http://www.fao.org/docrep/article/wfc/xii/0177-c2.htm.

BAUMEISTER (M.), CARDEW (M.), LINOT (M.), MADESCLAIRE (A.). - Forêts et paysages en Lorraine : cahiers de lecture. - Nancy : ONF Lorraine; CRPF Lorraine-Alsace, 2002. - 134 p.

BREMAN (P.). - Approche paysagère des actions forestières, guide à l'attention des personnels techniques de l'Office national des Forêts. - Fontainebleau : ONF-DTC, 1993. - 76 p.

BREMAN (P.). - Cahier de recommandations à l'usage des sylviculteurs pour une approche paysagère de la production en forêt morvandelle. - Dijon : Préfecture de la Région de Bourgogne, 1997. - 104 p.

BREMAN (P.), MOIGNEU (T.), LAVERNE (X.). - Directives paysagères pour la région Ile-de-France. - Fontainebleau : DTC-ONF, 1992. - 66 p.

CEMAGREF. - Reboisement et paysage. - Note technique $\mathrm{n}^{\circ} 45$. - Nogent-sur-Vernisson : Cemagref, 1981. - $112 \mathrm{p}$.

CRAMER (L.A.). - Changing Forest Service Values and Their Implications for Land Management. - Rural Sociology, vol. 58, $\mathrm{n}^{\circ}$ 3, 1993, pp. 475-491.

CRIGNON (C.), MULOT (A.-C.). - La Prise en compte du paysage dans la mise en œuvre des mesures agricoles et forestières au sein des DRAF et des DDAF. - Paris : ENGREF, 2001. - $43 \mathrm{p}$.

DASNOY (C.), MORMONT (M.), MOUGENOT (C.). - De la forêt au territoire, évolution du métier de forestier. - Environnement et Société, vol. 24, 2000, pp. 41-55.

DERF. - Conditions de financement, par le budget général de l'État, des projets d'investissements forestiers ou d'actions forestières à caractère protecteur, environnemental et social. Circulaire DERF/SDT/C2001-3010 du 07 mai 2001. - Paris : DERF 2001. - 43 p.

DERF. - Prise en compte du paysage dans la gestion forestière et les opérations de boisement. Circulaire/DERF/SDF/No3001. - Paris : Ministère de l'Agriculture - Sous-Direction de la Forêt, 1996. - 4 p.

DEUFFIC (P.). - Perception des impacts paysagers des actions forestières. - Ingénieries EAT, vol. 7, 1996, pp. 31-39.

DEUFFIC (P.), BARTHOD (C.). - Acquérir de nouvelles compétences environnementales par la formation professionnelle continue. In : XII Congrès forestier mondial, FAO, Québec, 2003. http://www.fao.org/docrep/article/wfc/xii/oo86-c4.htm. 
FREYTET (F.), RUMELHART (M.). - Les forestiers, fabricants de paysage. - Forêt-Entreprise, vol. 140, 2001, pp. 28-34.

GERNIGON (C.). - Un guide paysager pour la forêt limousine. - Limoges : ONF - Cellule Forêt-Paysage du Limousin, 2002. $-171 \mathrm{p}$.

GUAY (L.), GAGNON (E.). - Légitimité professionnelle et reconnaissance sociale : l'exemple des ingénieurs forestiers du Québec. - Sociologie et sociétés, vol. XX, n² 2, 1988, pp. 141-162.

JOUVENEL (G.), MASINGUE (B.). - Les Évaluations d'une action de formation dans les services publics. Paris : Les Éditions d’Organisation, 1994. - 207 p.

KENNEDY (J.), THOMAS (J.), GLUECK (P.). - Evolving forestry and rural development beliefs at midpoint and close of the 2oth century. - Forest Policy and Economics, vol. 3, 2001, pp. 81-95.

LUGINBÜHL (Y.). - Bilan et prospective de la politique paysagère de la DERF. Ministère de l'Agriculture. Versailles : École nationale supérieure du Paysage de Versailles, 1993. - 40 p.

LUGINBÜHL (Y.), BONTRON (J.-C.), CROS (Z.). - Méthodes pour des atlas de paysage, identification et qualification. - Paris : Ministère de l’Équipement - DAU, 1997. - 76 p.

MAAPAR. - L'Agriculture et la forêt dans le paysage. - Paris : MAAPAR/DERF, 2002. - 104 p.

ONF. - Guide des traitements des paysages. - Paris : ONF, 1999. - 64 p. (classeur avec fiches).

ONF. - Routes forestières : recommandations techniques. - Paris : ONF, 2000. - 140 p.

SCHMITHÜSEN (F.), KAZEMI (Y.). - Analyse des rapports entre les attitudes des gens envers la forêt et leurs attitudes envers la gestion forestière. - Journal forestier suisse, vol. 146, n० 4, 1995, pp. $247-264$.

WAGNER (R.G.), FLYNN (J.), GREGORY (R.), MERTZ (C.K.), SLOVIC (P.). - Acceptable practices in Ontario's forests: Differences between the public and forestry professionals. - New Forests, vol. 16, ${ }^{\circ}$ 2, 1998, pp. $139-154$.

APRÈs LES PRIORITÉS DU JOUR, LE PAYSRGE... ENOUÊTE SUR UN ENJEU FLOU POUR LES SERUICES FORESTIERS [Résumé]

Entre 1996 et 2000, le ministère de l'Agriculture a incité les agents intervenant en forêt privée à suivre des formations continues sur le thème du paysage. En 2002, il a souhaité faire le point sur les attentes et les difficultés que rencontrent encore les agents formés d'une part et les agents non formés d'autre part. L'enquête menée en 2002 auprès de 109 agents forestiers de DDAF, de CRPF et de SERFOB montre en effet que la prise en compte du paysage dans la gestion forestière reste très difficile. Le manque de motivation des propriétaires forestiers, l'absence de moyens incitatifs et le niveau de compétence des agents restent des obstacles majeurs. Néanmoins, les agents interrogés sont persuadés que les questions de paysage se poseront de plus en plus souvent aux acteurs du monde forestier. Nombre de ses agents envisagent donc d'enrichir leur connaissance dans ce domaine. L'accès à des ressources bibliographiques du type "guides techniques" leur paraît être un des meilleurs moyens d'y parvenir, bien avant celui de la formation continue.

\section{FIRST THE ImmEDIATE PAIORITIES, THEN THE LANDSCAPE - A SURUEY ON A DEUELOPMENT THAT REMAINS FUZZY FOR FORESTRY SERUICES [Hbstract]}

Between 1999 and 2000, the Ministry of Agriculture encouraged state foresters who operate with private forests to attend training sessions on landscape planning. In 2002, the Ministry made an assessment of the outstanding difficulties and problems facing state foresters, both trained and untrained. A survey was carried out among 109 employees. According to the survey, the most significant issues are forest owners' lack of motivation, inadequate subsidies and inadequate skills of state foresters. Respondents are nonetheless convinced that landscape will be a key issue in the near future. Many foresters therefore wish to improve their knowledge in this area. One of the best ways of achieving this, in their view, is to have access to technical guides and better circulation of the information, which they place well ahead of continuing education programmes. 